\title{
Performance Analyses of the Radio Orbital Angular Momentum Steering Technique Based on Ka-Band Antenna
}

\author{
Mingtuan Lin, ${ }^{1,2}$ Yue Gao, ${ }^{2}$ Peiguo Liu, ${ }^{1}$ and Zhiqing Guo ${ }^{3}$ \\ ${ }^{1}$ College of Electronic Science and Engineering, National University of Defense Technology, Changsha 410073, China \\ ${ }^{2}$ School of Electronic Engineering and Computer Science, Queen Mary University of London, London E1 4NS, UK \\ ${ }^{3}$ School of Marine Engineering, Jimei University, Xiamen 361021, China \\ Correspondence should be addressed to Mingtuan Lin; linmingtuan08@163.com
}

Received 5 April 2017; Revised 18 May 2017; Accepted 24 May 2017; Published 28 June 2017

Academic Editor: Sotirios K. Goudos

Copyright (C) 2017 Mingtuan Lin et al. This is an open access article distributed under the Creative Commons Attribution License, which permits unrestricted use, distribution, and reproduction in any medium, provided the original work is properly cited.

\begin{abstract}
The misalignment in the orbital angular momentum- (OAM-) based system would distort the radiation patterns of twisted beams carrying OAM, consequently making the OAM-based communication infeasible. To tackle the misalignment problem, a radio OAM steering technique based on a uniform circular array (UCA) is illustrated. Subsequently, simulations are conducted to explore the influence of the OAM steering on the OAM mode quality and transmission performance. Furthermore, UCAs working at Kaband with formulated feeding networks are designed and fabricated to analyze the performance of the OAM steering. The influences of OAM steering on mode quality and orthogonality are then evaluated in the experiment. Overall, the analyses of OAM steering technique are beneficial for the development of radio OAM study.
\end{abstract}

\section{Introduction}

The electromagnetic field carrying the spin angular momentum (SAM) known as the polarization had been widely studied in the past, while the other intrinsic physical property of the electromagnetic field, that is, orbital angular momentum, was not discovered until it was reported in Allen group's research [1] in 1992. OAM in the optical regime for application of imaging, microscopic particle, and communication [2, 3] had been then highly developed. However, investigations about the radio OAM had not been made until Bo Thide's group conducted the radio OAM experiment in [4], which elaborated another perspective of multiplexing, that is, OAM multiplexing for the present communication. In contrast to the planar wave, twisted beam with OAM mode has a helical wave front known as vortex. Benefiting from its peculiar properties such as the orthogonality of integer topological modes, large degrees of freedom, and rich phase information, OAM-based communication and radar techniques attract increasing attention.

To date, researches about radio OAM mainly lie on the twisted wave antenna design and OAM multiplexing.
Uniform circular array (UCA) with each element fed with the same amplitude but successive phase shift was raised in [5] to produce the twisted wavefront. Based on UCA, several types of antenna array were designed and tested in $[6,7]$. Spiral phase plate (SPP) [8], by adjusting the length of the plate along the azimuthal direction, is another major approach to create OAM. Combining concepts of SPP and array, a plate was designed and tested in [9] to produce mixed twisted beams at $94 \mathrm{GHz}$. Later, a design of an offset-fed reflection SPP to generate high purity OAM was reported in [10].

Meanwhile, OAM-based multiplexing has also attracted much attention. Experiment with two channels data modulated on two OAM modes was conducted in [11]. Subsequently, a 32-Gbit/s millimeter-wave link over 2.5 meters with OAM multiplexing at $28 \mathrm{GHz}$ was illustrated in [12]. Based on traveling circular-wave antennas [13], dual OAM modes multiplexing communication experiment at $60 \mathrm{GHz}$ was conducted in [14]. However, several studies demonstrated controversial voice about the OAM multiplexing. It was illustrated in [15] that OAM can be derived from multiple input and multiple out (MIMO) theory, implying that OAM is a subtopic of the MIMO technique rather 


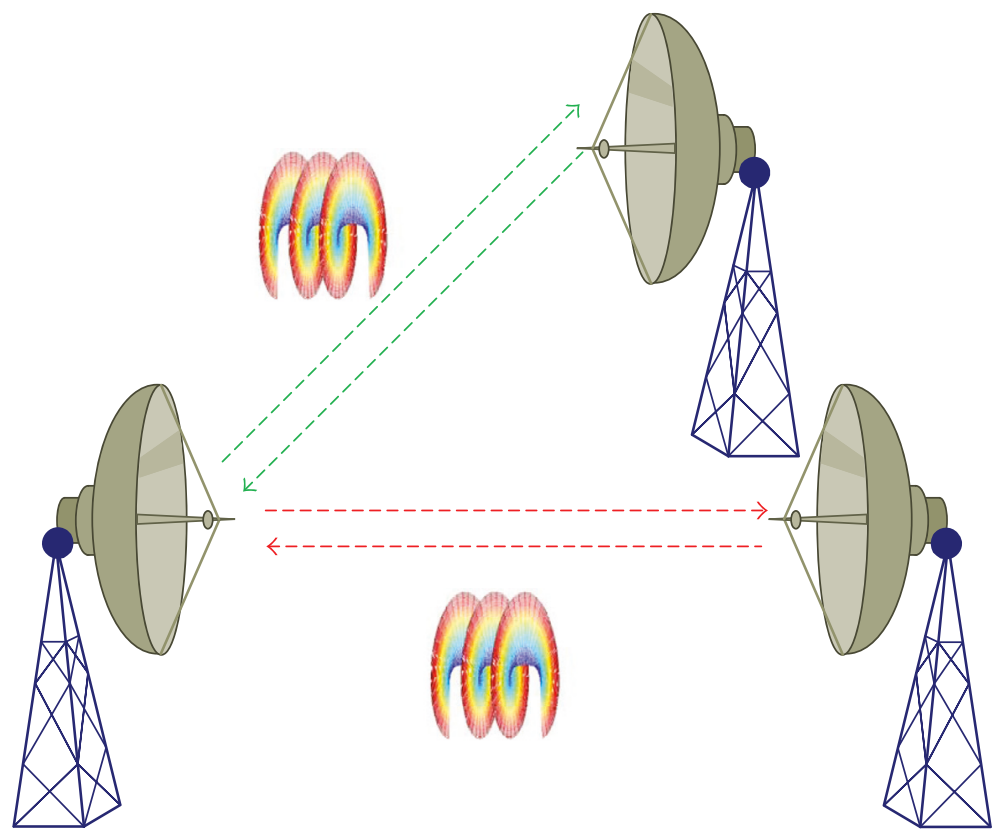

FIGURE 1: OAM-based communication system.

than a new regime and therefore cannot breakthrough the communication capacity. In addition, it was revealed in [16] that OAM multiplexing can only be applicable to short range distance communication due to the beam divergence. Regarding the controversy, it should be noted that OAM and MIMO have intersection when using UCA to produce OAM; however, they are absolutely two different domains. For instance, in OAM domain a higher OAM mode $(l>0)$ can be generated by a single antenna element, whereas in MIMO domain it requests more than $2 l$ antenna elements. It is admitted that OAM-based communication can not breakthrough the communication capacity, whereas, unlike MIMO-based system, OAM-based system does not require any complex signal processing [17] and channel information, which would be of significance for the low-complexity and high-speed communication at short range.

The application of radio OAM in radar regime $[18,19]$ also becomes a hot topic. Target detection model based on OAM was proposed in [20], which employed a UCA to produce twisted beams to illuminate objects in the far field, providing a new perspective to estimate azimuth information of targets without any relative moves or beams scanning required by traditional radars. To further increase resolution of the OAM-based radar technique, multiple signal classification (MUSIC) algorithm was introduced in our recent studies [21, 22], showing great resolution improvement and robustness enhancement.

The OAM-based communication system can be used in application where large amount data need to be exchanged such as the wireless server exchange center. However, this point-to-point system requires that the apertures of transmitter and receiver are strictly aligned to each other, as the red dash line shown in Figure 1. The experiment in [7] reported that the OAM-communication system was sensitive to the misalignment problem, which would degrade the capacity and needs to be solved. However, it challenges a lot for mounting or alignment where antennas of both sides are biased in a certain direction and the mechanical alignment is not easily available, such as the setup of antennas in roof of building or large size antenna mounting. Previous study [23] about twisted beams steering was based on a reflection phase modulated metasurface, which was utilized for simultaneously producing multiple OAM modes at $5.8 \mathrm{GHz}$. However, it does not refer to the solution of misalignment problem for OAM-based system. In addition, the horn antenna in the center would block part of reflected wave and cause scattering, therefore, affecting the quality of OAM mode. To avoid the blocking effects, this study proposes a UCA to realize the OAM steering technique, in order to solve the misalignment problem of OAM-based system. The aim of this study is to evaluate the misalignment problem and to analyze the performance of radio OAM steering technique. Our design is based on 8-element UCA with formulated feeding network to steer the twisted wave in an expected direction, which will enable the oblique transceiver sides to communicate with twisted beams as the green dash line shown in Figure 1. Given that radio OAM in millimeter wave or submillimeter wave can be beneficial for the fifth generation $(5 \mathrm{G})$ short range communication and the Ka-band has been selected as one of the future $5 \mathrm{G}$ spectrum candidate, the antenna and experiment are designed at $28 \mathrm{GHz}$. Overall, the main contributions of this paper include

(1) the design of UCA to realize radio OAM steering technique to solve the misalignment problem;

(2) analyses of OAM steering on OAM mode quality and transmission performance; 


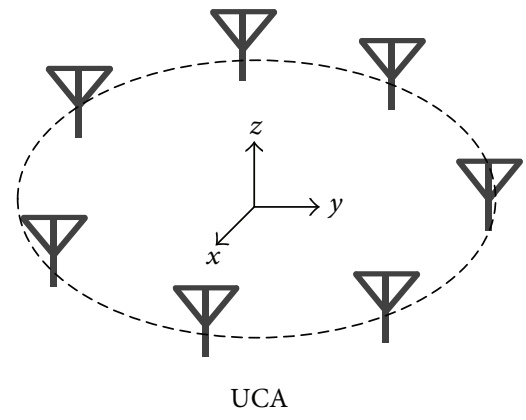

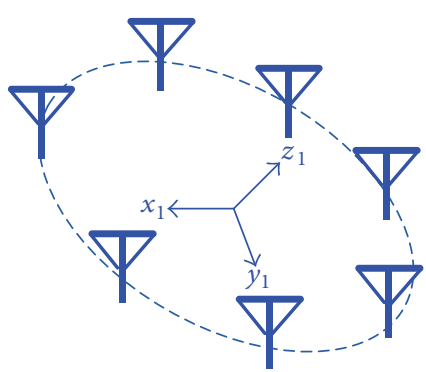

Equivalent UCA

FIgURE 2: UCA and the equivalent UCA.

(3) evaluation of distortion in the misaligned OAMbased system;

(4) experimental evaluation of radio OAM steering technique.

\section{Principle of Radio Orbital Angular Momentum Steering}

Before introducing the OAM steering, let us first review the traditional beamforming. By feeding the UCA in Figure 2 with the steering weight

$$
\begin{aligned}
& \mathbf{W}_{s} \\
& \quad=\left[e^{-j\left(2 \pi\left(\vec{d}_{1} \cdot \vec{k}_{0}\right) / \lambda\right)}, e^{-j\left(2 \pi\left(\vec{d}_{2} \cdot \vec{k}_{0}\right) / \lambda\right)}, \ldots, e^{-j\left(2 \pi\left(\vec{d}_{N} \cdot \vec{k}_{0}\right) / \lambda\right)}\right],
\end{aligned}
$$

the main beam of UCA can be steered in the expected direction $\left(\theta_{0}, \varphi_{0}\right)$, where $\vec{d}_{i}$ is the position vector of $i$ th element of UCA, $\theta_{0}$ and $\varphi_{0}$ are the elevation and azimuth angle respectively, $\vec{k}_{0}=\left[\sin \theta_{0} \cos \varphi_{0}, \sin \theta_{0} \sin \varphi_{0}, \cos \theta_{0}\right]^{T}$ is the unit propagation vector, and $\lambda$ is the wavelength. The radiation pattern of the UCA array fed with (1) can be conceived as the radiation pattern of an equivalent UCA as shown in Figure 2 with uniform feeding. $x-y-z$ refers to the coordinate of the UCA, while $x_{1}-y_{1}-z_{1}$ is the coordinate of the equivalent UCA. Under $x-y$ - $z$ coordinate, $z_{1}$-axis points to $\left(\theta_{0}, \varphi_{0}\right)$.

It was proved in [5] that twisted beams can be generated by a UCA. Suppose that a UCA consists of $N$ elements, with each element fed with the same amplitude but a successive phase difference $\Delta \phi=2 \pi l / N$ between each other, where $l$ is OAM mode. Therefore, the weight of array to produce twisted beams with OAM mode $l$ can be expressed as follows:

$$
\mathbf{W}_{l}=\left[1, e^{j(2 \pi l / N)}, \ldots, e^{j(2 \pi(N-1) l / N)}\right] .
$$

By combining $\mathbf{W}_{s}$ and $\mathbf{W}_{l}$, the twisted beam can be steered in a specific direction using a UCA, with $\mathbf{W}_{s}$ responsible for the beam steering and $\mathbf{W}_{l}$ in charge of generation of OAM mode $l$. Therefore, the total weight $\mathbf{W}$ with the formulation

$$
\mathbf{W}=\mathbf{W}_{s} \otimes \mathbf{W}_{l}
$$

can be employed to generate mode $l$ twisted beam in the steering direction $\left(\theta_{0}, \varphi_{0}\right)$, where $\otimes$ denotes the dot-product. By feeding the UCA in Figure 2 with weight $\mathbf{W}$, it can be approximately regarded as the equivalent UCA fed with $\mathbf{W}_{l}$. As the size of equivalent UCA becomes smaller, the beam width of the doughnut shape produced by radio OAM steering would be wider than that of normal way to generate OAM.

\section{Simulation Analyses of Radio Orbital Angular Momentum Steering}

Based on the formulated feeding network in the Section 2, simulations are conducted in this section to investigate how the steering azimuth angle and elevation angle affect different OAM modes. Suppose that a UCA working at $28 \mathrm{GHz}$ has $N=8$ elements, with a radius of $20 \mathrm{~mm}$. Perpendicular to the steering direction, the observed area is $d=0.5 \mathrm{~m}$ far from the UCA, with a size $0.8 \mathrm{~m} \times 0.8 \mathrm{~m}$.

To further analyze the distortion, OAM mode decomposition [24] is utilized to calculate OAM spectrum of each mode. Here we extend the decomposition to the whole observed area as follows:

$$
\widetilde{\Phi}_{r_{0}, l}=\frac{1}{2 \pi} \int_{-\pi}^{\pi} \Phi\left(r_{0}, \varphi\right) e^{-j l \varphi} d \varphi,
$$

where $\widetilde{\Phi}_{r_{0}, l}$ is the OAM spectrum of mode $l$ at radical distance $r_{0}$ and $\Phi\left(r_{0}, \varphi\right)$ is observed phase distribution along $\varphi$. Integral is normally conducted in the doughnut shape circle, which is of most concern, but here all areas are involved. Through this extended OAM decomposition method, it is easy to gain the 2D OAM spectrum.

3.1. Influence of Steering Elevation Angle on OAM Mode. To evaluate the influence of the steering elevation angles on OAM mode, three twisted beams carrying OAM mode 2 are steered in three directions, with $\theta_{0}=0^{\circ}, 30^{\circ}, 60^{\circ}$ and all the azimuth angles $\varphi_{0}=60^{\circ}$. For $\theta_{0}=0^{\circ}$, it means that the OAM mode is generated in the normal way without using the OAM steering technique. The radiation patterns for three steering angles are given in Figure 3. For the normal way to generate OAM, the helical phase profile and doughnut shape intensity pattern can be clearly observed in Figures $3(\mathrm{a})$ and $3(\mathrm{~d})$, respectively. From the OAM spectrum in 


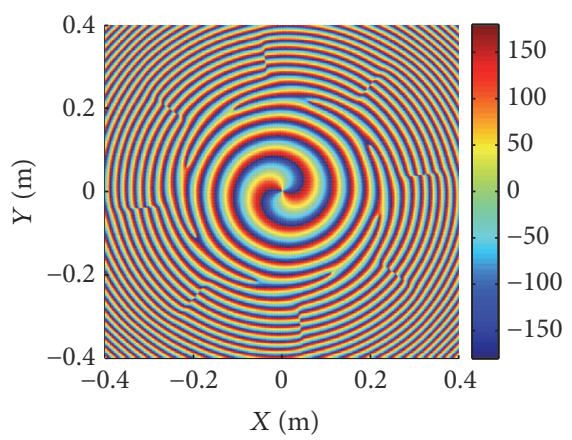

(a)

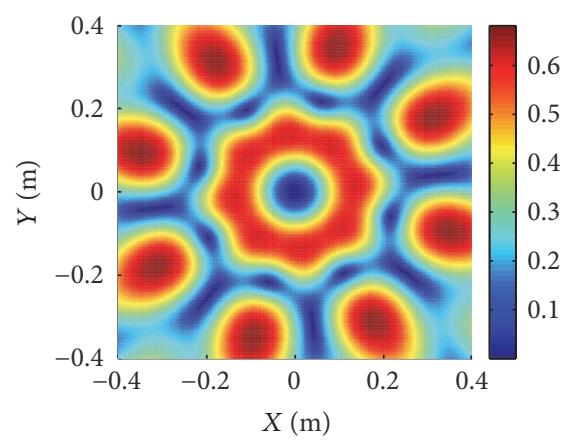

(d)

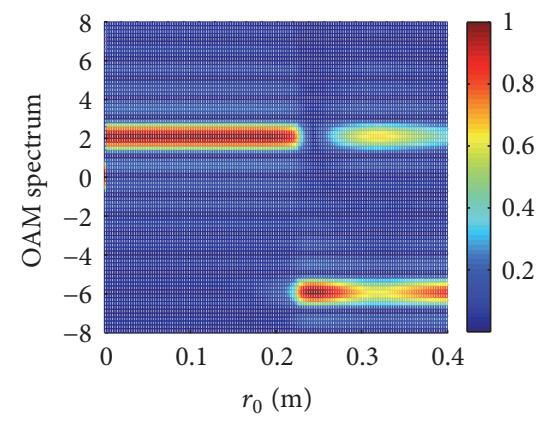

(g)

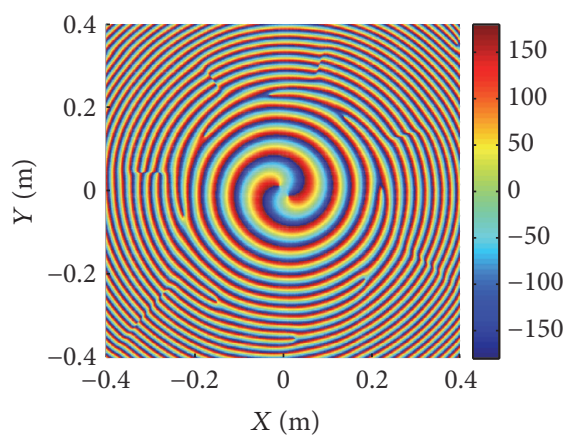

(b)

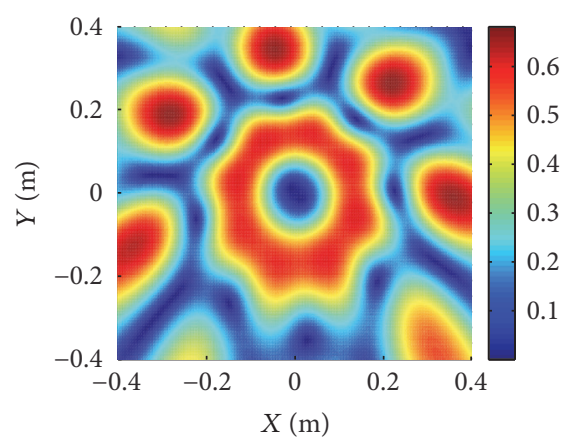

(e)

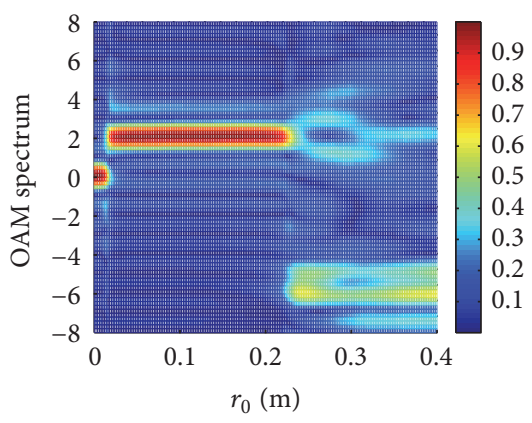

(h)

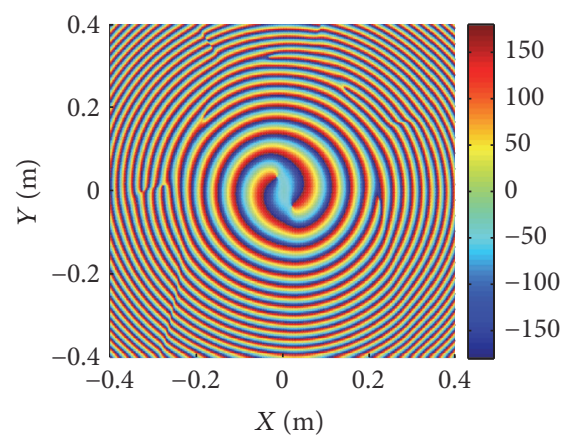

(c)

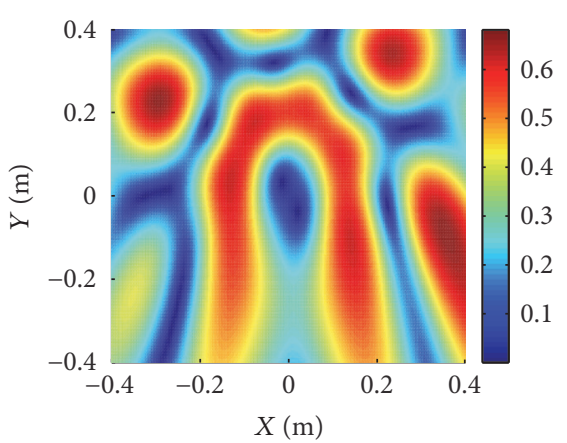

(f)

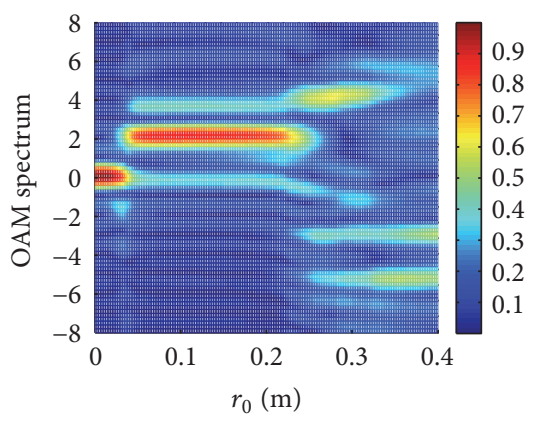

(i)

Figure 3: Phase profiles (a, b, c), intensity patterns ( $d, e, f)$, and the OAM spectrum $(g, h, i)$ after utilizing radio OAM steering in three different directions. $(\mathrm{a}, \mathrm{d}, \mathrm{g}),(\mathrm{b}, \mathrm{e}, \mathrm{h})$, and $(\mathrm{c}, \mathrm{f}, \mathrm{i})$ refer to the steering angles $\left(0^{\circ}, 60^{\circ}\right),\left(30^{\circ}, 60^{\circ}\right)$, and $\left(60^{\circ}, 60^{\circ}\right)$, respectively.

Figure $3(\mathrm{~g})$, pure OAM mode 2 can be seen within the main beam, that is, the first doughnut shape, beyond which the other OAM mode -6 is induced, therefore contaminating the purity of OAM mode. This contamination is because of the nonorthogonality between mode -2 and $-2+8$ for UCA with small $N$. With respect to the steering angle $\left(\theta_{0}, \varphi_{0}\right)=$ $\left(30^{\circ}, 60^{\circ}\right)$, the OAM spectrum in Figure $3(\mathrm{~h})$ shows that the OAM mode 2 degrades to 0 in the central area $\left(r_{0}<\right.$ $0.02 \mathrm{~m})$, consistent with the phase profile in Figure 3(b), and mode 4 is induced within the main beam $r_{0}<0.2 \mathrm{~m}$ although mode 2 still plays the dominant role, beyond which mode 2 is severely contaminated. Moreover, the doughnut shape intensity pattern in Figure 3(e) is a little bit distorted. More severe degradation and contamination within the main beam can be observed when the steering angle is $\left(60^{\circ}, 60^{\circ}\right)$, and the doughnut shape intensity pattern degrades to an open " $U$ " shape. Comparing the results of three different steering elevation angles, it can be found that the larger the steering elevation angle, the more severe the degradation and contamination problem the radio OAM steering technique suffers from.

3.2. Influence of Steering Azimuth Angle on OAM Mode. Keeping the elevation angle as a constant and changing azimuth angle, the simulation of radio OAM steering with mode 2 at $\left(30^{\circ}, 30^{\circ}\right)$ is conducted as a comparison with OAM steering at $\left(30^{\circ}, 60^{\circ}\right)$. The helical phase profile, doughnut shape, and the OAM spectrum in Figure 4 show almost the same performance as that in Figures 3(b), 3(e), and 3(h), implying that the steering azimuth angle does not affect the OAM mode quality for radio OAM steering technique. Therefore, in practical application, what we concern most should be the influence of the elevation angle rather than the azimuth angle. 


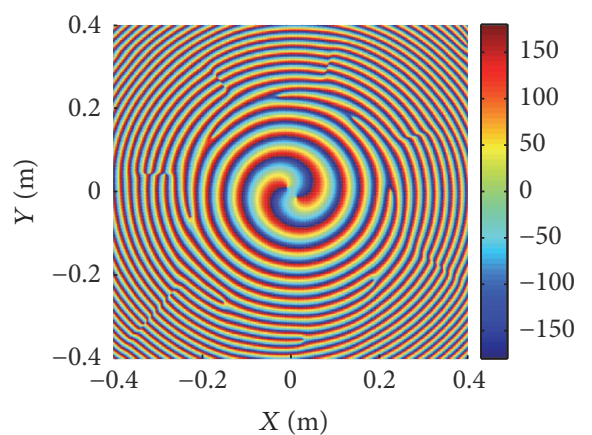

(a)

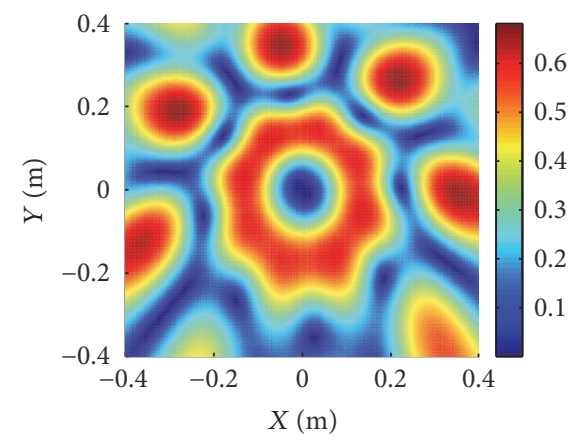

(b)

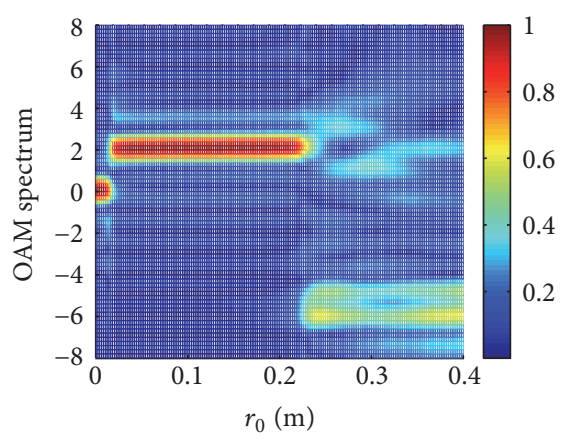

(c)

FIgURE 4: (a) Phase profile, (b) intensity pattern, and (c) the OAM spectrum after utilizing radio OAM steering at $\left(30^{\circ}, 30^{\circ}\right)$.

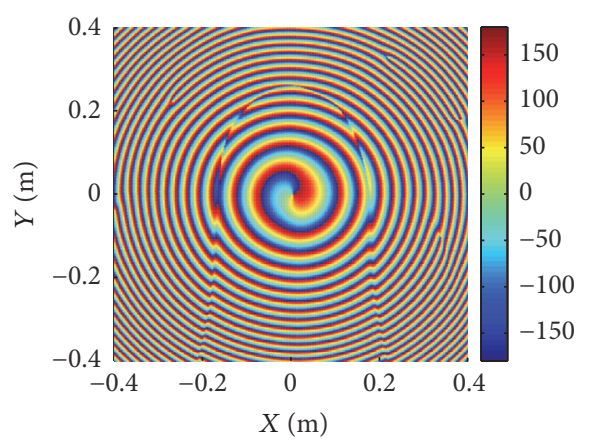

(a)

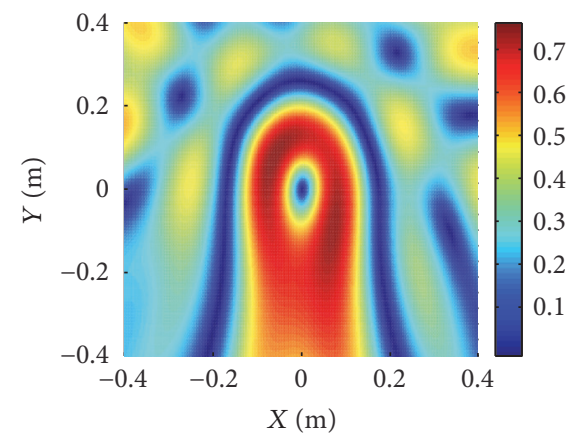

(b)

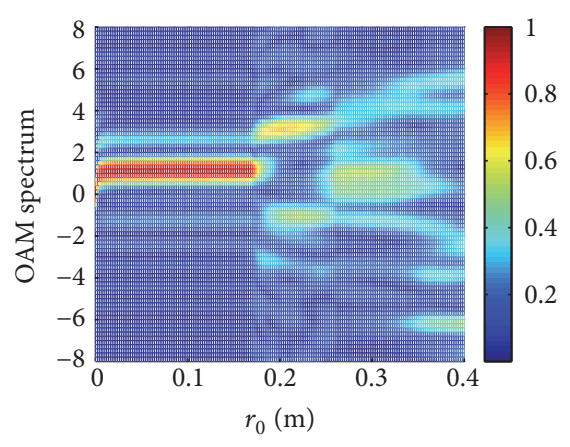

(c)

Figure 5: (a) Phase profile, (b) intensity pattern, and (c) the OAM spectrum after utilizing radio OAM steering to generate mode 1 at $\left(60^{\circ}, 60^{\circ}\right)$.

3.3. Influence of Radio OAM Steering Technique on Different OAM Modes. As analyzed in previous two subsections, the OAM mode quality is affected by the OAM steering elevation angle. The influence of radio OAM steering technique on different OAM modes is explored in this subsection. By configuring the simulation with the same steering angle $\left(60^{\circ}, 60^{\circ}\right)$ as that in Figure 3, mode 1 is chosen as comparison with mode 2 . From Figure 5 , it can be seen that both the helical phase profile and intensity pattern in Figures 5(a) and 5(b) are distorted, and the OAM spectrum in Figure 5(c) presents degradation and contamination problems similar to that in Figure 3. In comparison with Figures 3(c), 3(f), and 3(i) and Figure 5, the intensity pattern shape of mode 1 does not degrade to an open " $U$ " shape and smaller central area is affected by the degradation problem, meanwhile the contamination influence is much less than that of mode 2 . It can be found that the higher OAM mode suffers from the more severe degradation and contamination problem when using the radio OAM steering technique.

3.4. Influence of Radio OAM Steering Technique on OAMBased Communication. To some extent, OAM steering technique causes distortion for different OAM modes. To evaluate the influence of mode distortion on communication transmission, simulations of total bit error rates (BERs) of all modes by using the nonsteering OAM wave and the steering OAM wave are conducted in this subsection. An 8element UCA with a radius of $2 \lambda$ is used as the transmitter, while the receiver UCA usually has a larger aperture to ensure good communication. Here the radius of receiver is $5 \lambda$, and the receiver is located one Rayleigh distance from the transmitter. The transmitter and receiver are aligned at steering directions. 7 different channel signals are modulated on 7 OAM modes using the amplitude-shift keying (ASK) modulation. Each channel consists of $10^{7}$ bits data. The receiver demodulates the incoming signals to 7 channels using the OAM demultiplexing method. The data of each channel is then obtained. In comparison with the original transmitted code, the total BER of 7 channels by OAM steering technique at different elevation angles can be achieved, which are shown in Figure 6. It can be seen that OAM-based communication needs high signal to noise (SNR) level to ensure low BER. To achieve $10^{-4} \mathrm{BER}, \mathrm{SNR}$ should be at least $32 \mathrm{~dB}$ for normal OAM-based communication. Under the same SNR level, OAM steering with larger elevation angles leads to higher communication BER, due to the more severe distortion of OAM modes. To achieve the same BER level $\left(10^{-4}\right)$ as the normal OAM-based communication, OAM steering with elevation angle $30^{\circ}$ requires SNR around $39 \mathrm{~dB}$, $7 \mathrm{~dB}$ higher than that of normal OAM-based communication.

\section{Antenna Design for Radio OAM Steering}

The analyses of the radio OAM steering technique in Section 3 show good qualities of OAM modes 1 and 2 for elevation angles no larger than $30^{\circ}$, which is of significance 
TABLE 1: The feeding phases and amplitudes of each patch for different antenna arrays.

\begin{tabular}{|c|c|c|c|c|c|c|c|c|}
\hline \multirow{2}{*}{ Patch } & \multicolumn{2}{|c|}{ OAM1 } & \multicolumn{2}{|c|}{ OAM2 } & \multicolumn{2}{|c|}{ OAM1_1 } & \multicolumn{2}{|c|}{ OAM2_1 } \\
\hline & Phase $\left({ }^{\circ}\right)$ & $\operatorname{Amp}(\mathrm{dB})$ & Phase $\left({ }^{\circ}\right)$ & $\operatorname{Amp}(\mathrm{dB})$ & Phase $\left({ }^{\circ}\right)$ & $\operatorname{Amp}(\mathrm{dB})$ & Phase $\left({ }^{\circ}\right)$ & $\operatorname{Amp}(\mathrm{dB})$ \\
\hline 1 & 0 & -10.15 & 0 & -10.13 & 0 & -10.4 & 0 & -10.14 \\
\hline 2 & $45-0.4$ & -10.4 & $90-0.2$ & -10.32 & $-21.5+0.1$ & -10.1 & $23.4-0.6$ & -10.43 \\
\hline 3 & $-90+0.4$ & -10.34 & $180+0.2$ & -10.46 & -28 & -10.4 & $62-0.6$ & -10.37 \\
\hline 4 & $-45-0.3$ & -9.98 & $-90-0.2$ & -10.03 & $-94.5-0.5$ & -10.1 & $40.4+4$ & -10.13 \\
\hline 5 & 0 & -10.15 & $180-0.6$ & -9.94 & $-173+0.2$ & -9.9 & -6.8 & -10.06 \\
\hline 6 & $45-0.4$ & -10.4 & $-90+0.5$ & -10.33 & $-75.3-0.2$ & -10.4 & $149.7+0.2$ & -10.43 \\
\hline 7 & $-90+0.4$ & -10.34 & $180-0.6$ & -10.35 & 21.1 & -10.4 & $-68.9+0.2$ & -10.5 \\
\hline 8 & $-45-0.3$ & -9.98 & $-90+0.1$ & -9.96 & $177.7-0.2$ & -10.2 & $132.7-0.3$ & -9.85 \\
\hline
\end{tabular}

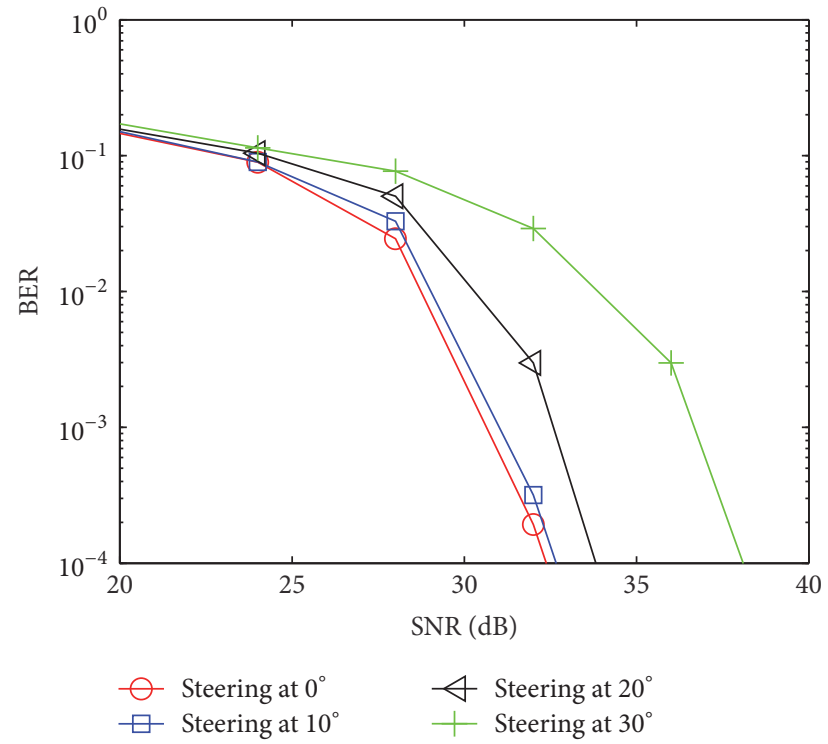

FIGURE 6: BER against SNR for different steering angles.

for misalignment OAM-based system. Prior to use of OAM steering technique, the misalignment information should be known in advance, which can be realized through reconfigurable phased antenna array. The reconfigurable feeding network can not only obtain the misaligned direction of the transmitter and receiver but also provide a dynamic control of the OAM steering. The misalignment information should be transmitted to each other in a traditional communication way before the OAM-based communication commences. For simplicity, we use the microstripe lines as the feeding network to verify the OAM steering technique. A UCA working at Kaband $(28 \mathrm{GHz})$ with $x$-polarization is designed as shown in Figure 7. The layout of this UCA contains two major parts, namely, 8 patch antenna units and the microstrip line feeding network. Having low electronic loss in high frequency band, Rogers 5880 PCB is chosen as the substrate, with dielectric constant $\varepsilon_{r}=2.2$ and thickness $h=0.508 \mathrm{~mm}$. The $3.4 \mathrm{~mm} \times 3.4 \mathrm{~mm}$ patch unit is optimized to have the resonant frequency of $28 \mathrm{GHz}$. To reduce the power differences of those 8 patch antennas, Wilkinson power divider in the dashed square is implemented among every two adjacent units. With compact space, T-junctions in the dashed circles rather than the Wilkinson power dividers are employed to connect each part. The phase of each unit is controlled by adjusting the length of feeding line to the patch, that is, $x_{i}(i \in$ $[1,8])$, which can be calculated according to the formulation of the feeding weights in (3). The radius of the array is $20 \mathrm{~mm}$.

Two UCAs with the proposed feeding weights are designed to generate OAM modes 1 and 2 in the same steering direction $\left(30^{\circ}, 60^{\circ}\right)$. The two UCAs are denoted by OAM1_1 and OAM2_1, respectively. As comparison, UCAs denoted by OAM1 and OAM2 are also designed to produce OAM modes 1 and 2 in the normal direction. By comparing the performance of these four UCAs, the influence of radio OAM steering on OAM mode can be verified. The line lengths to 8 patch units in the four UCAs are optimized to obtain the feeding weight as required in (3). Given that the polarization of patches $1,2,7$, and 8 is opposite with patches $3,4,5$, and 6 , a $180^{\circ}$ phase compensation should be considered in the design of feeding network. Table 1 shows the designed phase and amplitude for each patch, which are optimized at $28 \mathrm{GHz}$. The denotations - and + in the phase column refer to the difference between the theoretical and the optimized feeding, from which it can be observed that the phase differences are within $1^{\circ}$. The ideal amplitude for each patch passing through 3 power dividers should be $-9 \mathrm{~dB}$. Compared to the theoretical result, less than $1.5 \mathrm{~dB}$ insert losses are observed. And each array exhibits good amplitude consistence for patches, with less than $0.6 \mathrm{~dB}$ power difference. The wellmatched amplitude and phase ensure the performance of twisted beam generation and steering.

The radiation patterns of antennas OAM1_1 and OAM2_1 with the optimized feeding are simulated by CST and depicted in Figure 8. From the dashed lines we can observe that both twisted beams point to the steering direction as predicted. Specifically, the radiation pattern of OAM mode 1 in Figure 8(a) preservers a typical doughnut shape of twisted beam, while the pattern of OAM mode 2 exhibits a " $U$ " shape in the steering direction in Figure 8(b). It is noted that in Section 3 the intensity shape degradation does not appear for elevation angle $30^{\circ}$. However, those simulations are based on the ideal radiation pattern. In practical design, the feeding transmission line network and the connector also affect the radiation pattern, therefore causing the degradation to open " $U$ " shape for elevation angle $30^{\circ}$. As shown in Figure 9, the fabricated antennas have compact sizes. The flexible $2.92 \mathrm{~mm}$ 


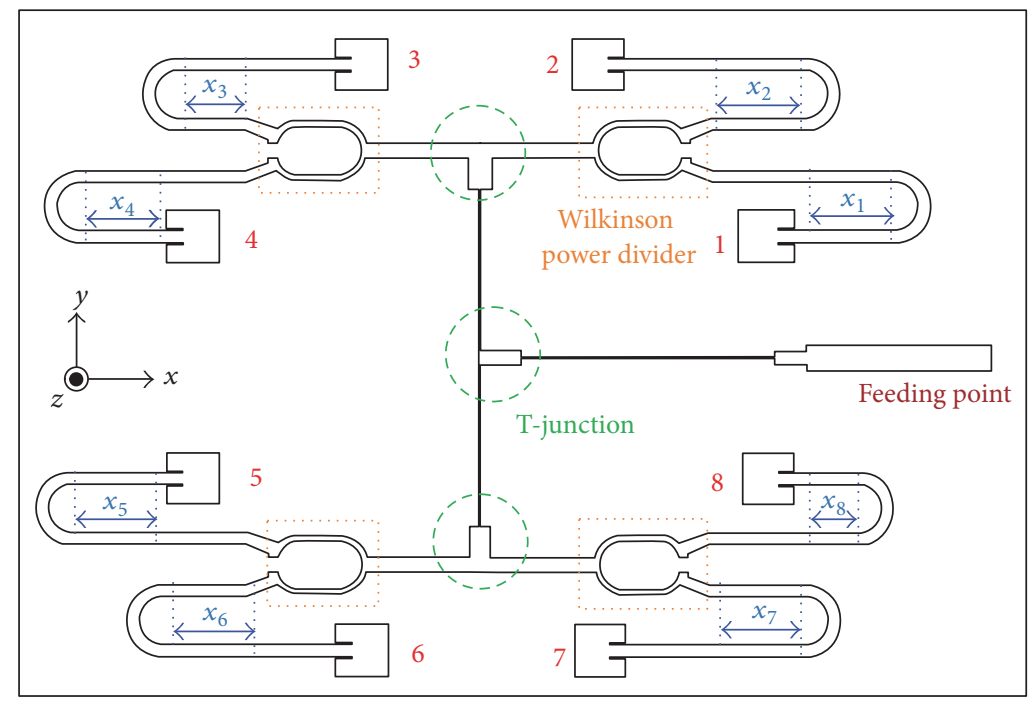

FIGURE 7: Layout of UCA and feeding network.

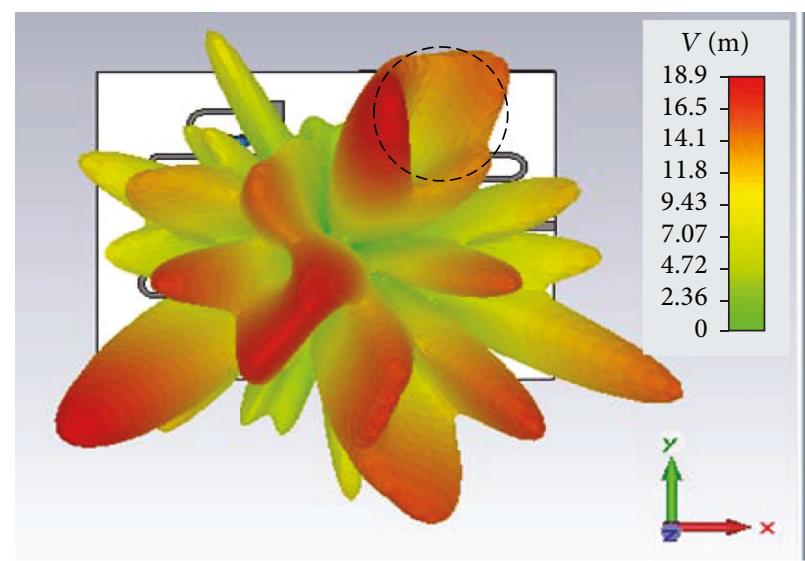

(a)

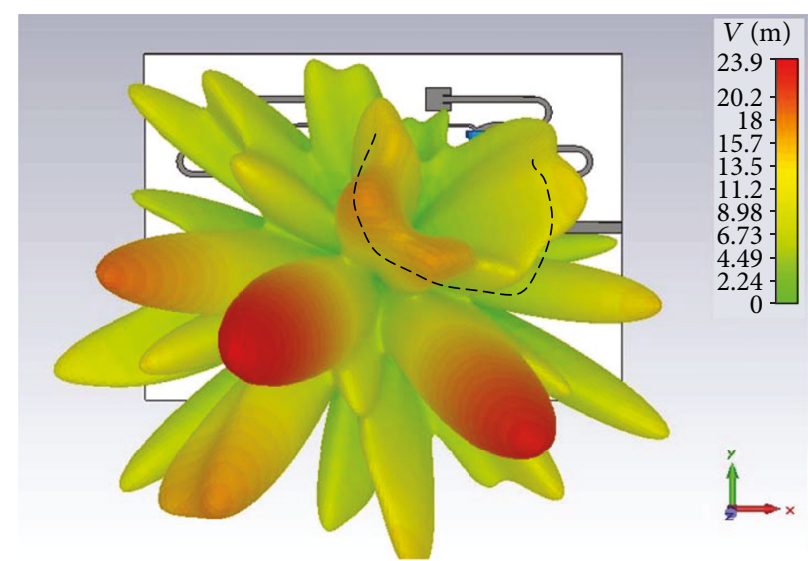

(b)

FIGURE 8: Radiation pattern of twisted wave steering antennas for (a) OAM mode 1 and (b) OAM mode 2.

end-launch connector is implemented in the design, which does not need soldering processing and can be easily installed and disassembled. $S_{11}$ for all antennas are measured and drawn in Figure 10, presenting low reflection with less than $-10 \mathrm{~dB}$ from $27 \mathrm{GHz}$ to $30 \mathrm{GHz}$. Particularly, $S_{11}$ at $28 \mathrm{GHz}$ are below $-15 \mathrm{~dB}$, showing good impedance matching of the arrays.

\section{Experimental Performance Analyses of the Radio OAM Steering Technique}

In this section, the distortion of OAM-based misaligned system is first experimentally evaluated. Subsequent experimental analyses of radio OAM steering technique to solve the misalignment problem are conducted.

5.1. Measurement Scenario and Setup. The transmitter antenna needs to be rotated first to construct the accurate misalignment condition. To realize it, a camera rotation platform with $2 \mathrm{D}$ rotation function is employed in the following experiment, which can simultaneously adjust the elevation and azimuth angles as shown in Figure 11(a). The installation of the antenna in the platform is illustrated in Figure 11(b). The plastic spacers are used to keep a distance between the antenna and the platform to avoid the scattering from the part of metal in the platform.

To evaluate the distortion of misaligned system and the twisted wave steering performance, three different setups for the transmitter and the receiver are made as shown in Figure 12. Aligned system in the first setup in Figure 12(a) is used to measure the radiation pattern and phase profile of antennas OAM1 and OAM2 to generate OAM modes 1 and 2 , whereas for the second setup, the same antennas are tested but in a misaligned configuration. To observe the distortion of misalignment, the antennas OAM1 and OAM2 are rotated $\left(30^{\circ}, 60^{\circ}\right)$ as shown in Figure 12(b). By comparing results 


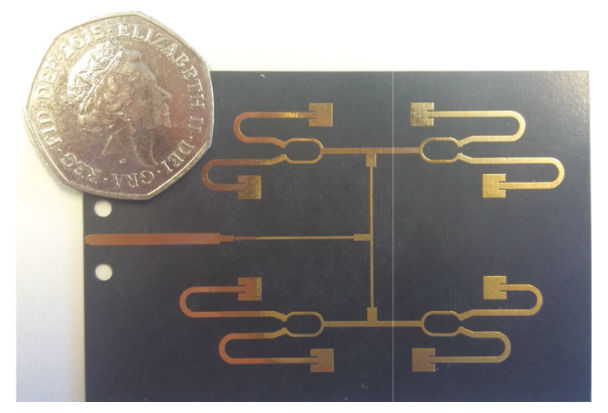

(a)

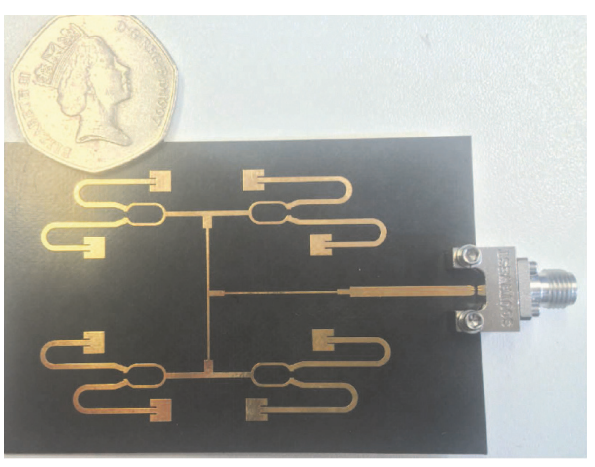

(b)

FIGURE 9: The fabricated antenna (a) without connector and (b) with connector.

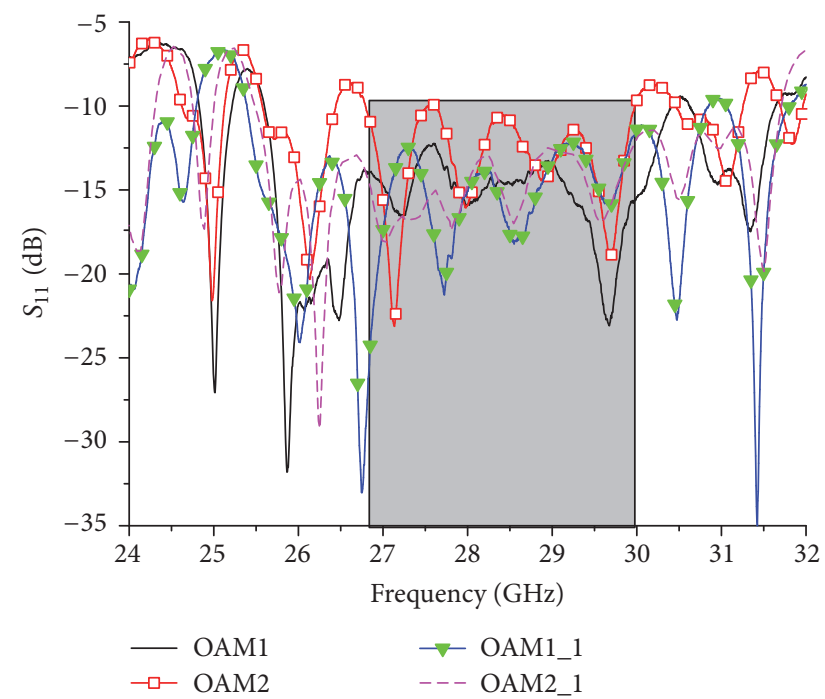

FIGURE 10: Measured reflection coefficients $S_{11}$ for all antennas.

of these two setups, the distortion of the misaligned OAMbased system can be directly obtained. In the third setup, two OAM steering antennas OAM1_1 and OAM2_1 are employed in the misaligned system to steer the twisted beams to solve the misalignment problem.

The setup of the experiment is shown in Figure 13(a), where a $2 \mathrm{D}$ scanner from Near Field Systems Inc. (NSI), $d=0.5 \mathrm{~m}$ far from the transmitter antennas, is employed to measure the intensity and phase profile. The receiver, using a probe either with horizontal or vertical polarization, and the transmitter are connected through a PNA-X network. With the control of the NSI scanner software, the radiation pattern of the measuring area can be obtained. Figure 13(b) is the picture of the setup of experiment.

5.2. OAM Mode Distortion of Misalignment. The phase profiles and intensity patterns of the first setup in a $0.3 \mathrm{~m} \times$ $0.3 \mathrm{~m}$ measuring area that can cover the main beams are recorded in Figures 14(a), 14(e), 14(b), and 14(f), where perfect helical phase profiles of OAM modes 1 and 2 can be clearly observed with doughnut shape intensity patterns. The field characteristics of arrays under the second misaligned setup are measured and presented in Figures 14(c), 14(g), $14(\mathrm{~d})$, and $14(\mathrm{f})$. A larger area $(0.4 \mathrm{~m} \times 0.4 \mathrm{~m})$ is selected as observation to have a full view of radiation pattern. Since the polarization of the probe in the NSI scanner can only be horizontal or vertical, the polarization of the transmitter under misaligned setup can not match the receiver well. To mitigate the influence caused by the mismatch of the polarization, horizontal polarization probe, matching better than vertical polarization, is chosen to measure the field. No helical phase profiles or doughnut shape patterns can be observed in the misaligned setup, showing the severe distortions of OAM modes. Obviously, the distorted OAM modes make the misaligned OAM-based system infeasible in practical application.

5.3. Performance Analyses of Radio OAM Steering Technique. Under misaligned setup, to retain good-quality twisted beams for receivers, antennas OAM1_1 and OAM1_2 generating OAM modes 1 and 2 in the steering direction perpendicular to the receiver are employed in the third setup experiment as shown in Figure 12(c). Excellent helical phase profile and doughnut shape intensity of OAM mode 1 measured with the horizontal polarization probe can be seen from the radiation pattern in Figures 15(a) and 15(e), while twisted wave with OAM mode 2 suffers from a minor distortion, with good helical wavefront but a "U" shape intensity pattern as shown in Figures 15(b) and 15(f), in an agreement with the simulated radiation pattern in Section 4. It can be found that the influence on OAM mode 2 is larger than mode 1 when the radio OAM steering technique is employed, consistent with the simulation analyses in Section 3. No blocking effects can be observed for the proposed radio OAM steering technique. Overall, the results, exhibiting good performance of OAM modes in the steering direction, imply that the twisted beam steering technique is feasible to retain the radio vortex for misaligned system. The radiation pattern measured with the vertical polarization probe under third setup is shown in Figures 15(c), 15(g), 15(d), and 15(h). Compared to the result measured with horizontal polarization probe, helical phase profile and the doughnut shape of mode 1 worsen, while for 


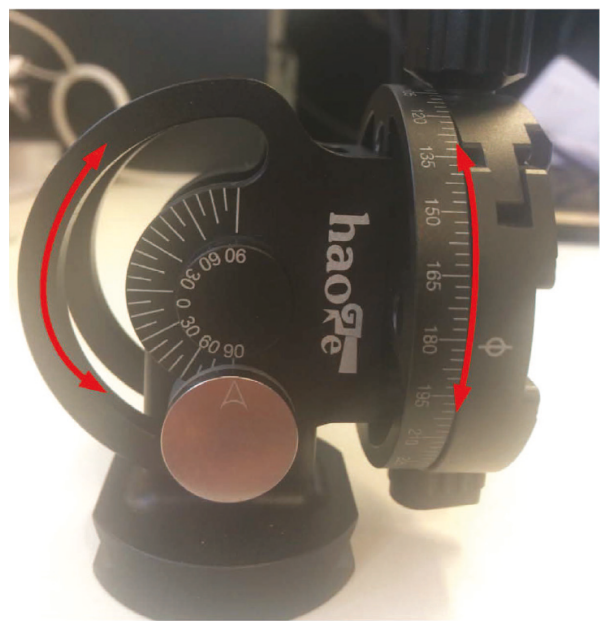

(a)

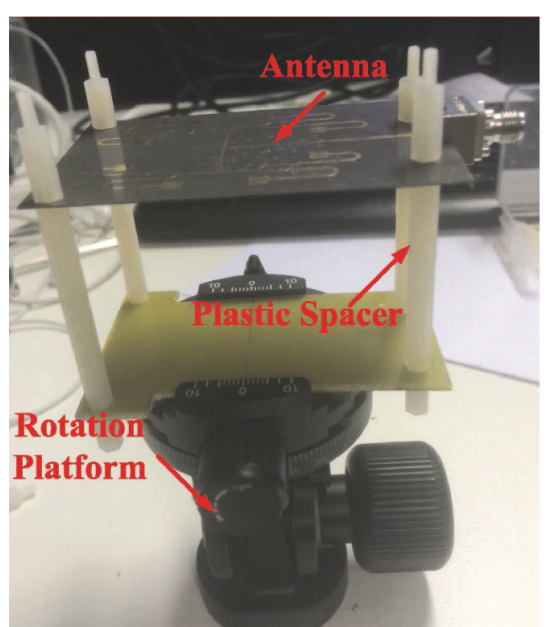

(b)

FIGURE 11: (a) The rotation platform and (b) the installation of antenna in the platform.

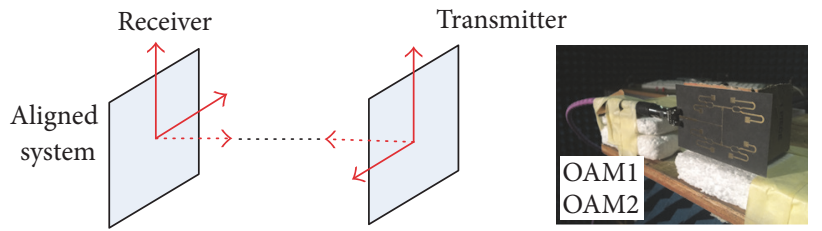

(a)
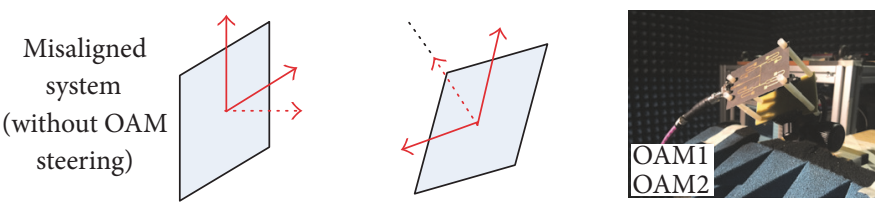

(b)
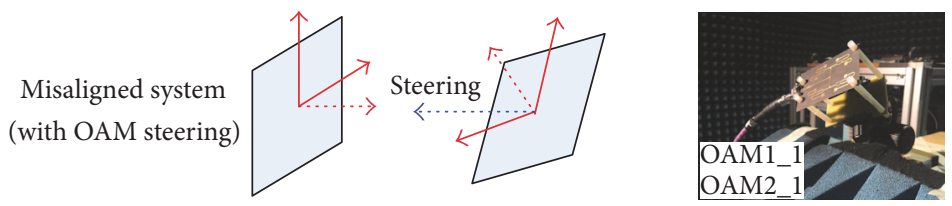

(c)

FIGURE 12: Experiment setup between the transmitter and receiver. (a) The aligned system, (b) misaligned system, and (c) misaligned system with twisted beam steering antennas.

mode 2 the properties of OAM can hardly be recognized. Obviously, the mismatch of polarization seriously affects the quality of twisted beams for the radio OAM steering technique. To realize the twisted beam steering technique, well-matched polarization between transmitter and receiver should be concerned.

Influence of OAM mode orthogonality after steering is also investigated in this subsection. Two UCAs are installed on the wooden board with a distance of $r=0.9 \mathrm{~m}$ as shown in Figure 16. For nonsteering OAM case, antennas are aligned in their normal directions, while for steering OAM waves, the normal directions of transmitter and receiver are misaligned and needs to be realigned using the OAM steering technique. Based on the fabricated antennas, the transmission coefficients of different OAM modes are conducted separately, namely, one mode by one mode rather than by multiplexing of all modes together. The transmission coefficients plotted in Figure 16 show that there is a $22 \mathrm{~dB}$ isolation of both modes at the central frequency $(28 \mathrm{GHz})$ for nonsteering OAM waves, demonstrating good orthogonality. As for the OAM steering waves with elevation angle $30^{\circ}$, the transmissions coefficients present a $18 \mathrm{~dB}$ isolation for these two modes, also showing 


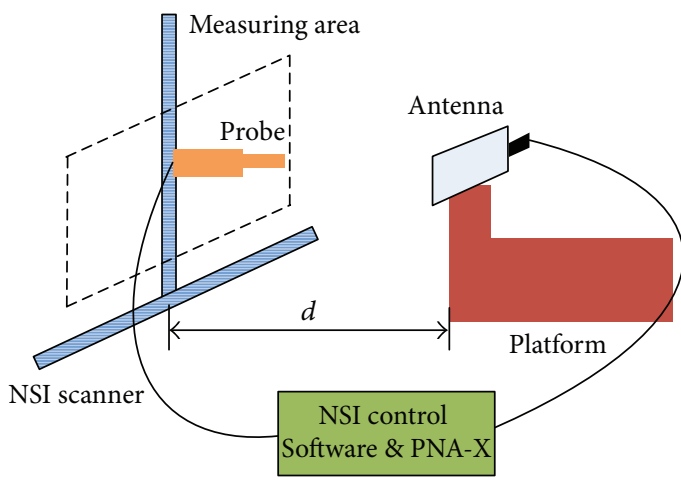

(a)

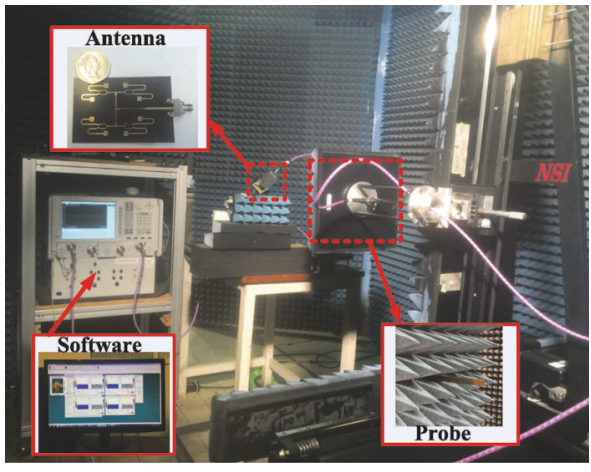

(b)

FIGURE 13: (a) Scheme of the measurement setup and (b) the picture of the measurement.

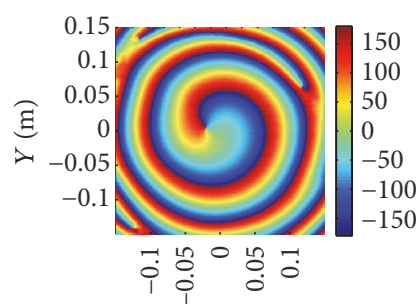

$X(\mathrm{~m})$

(a)

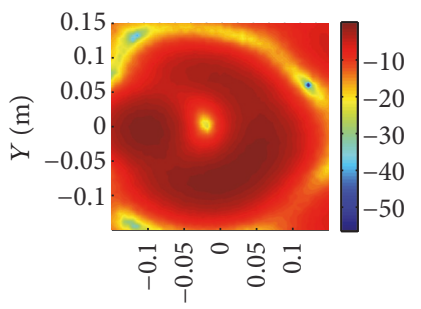

$X(\mathrm{~m})$

(e)

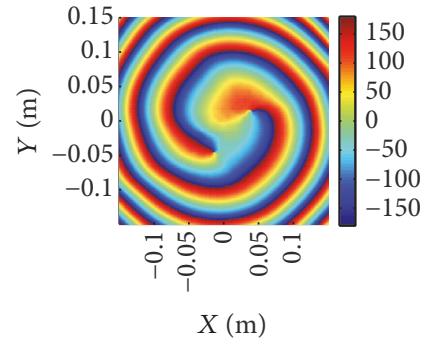

(b)

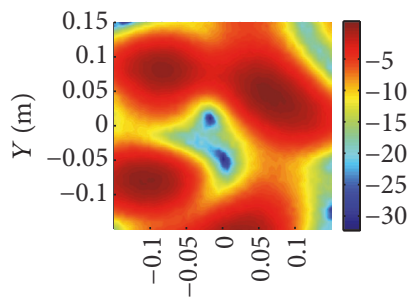

$X(\mathrm{~m})$

(f)

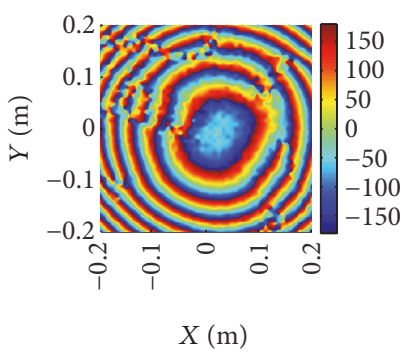

(c)

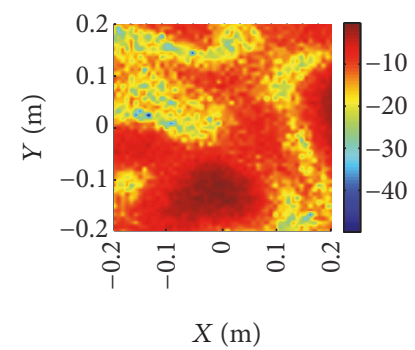

(g)

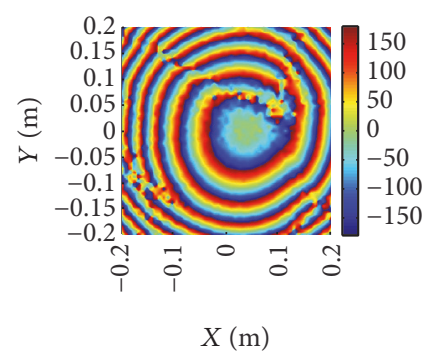

(d)

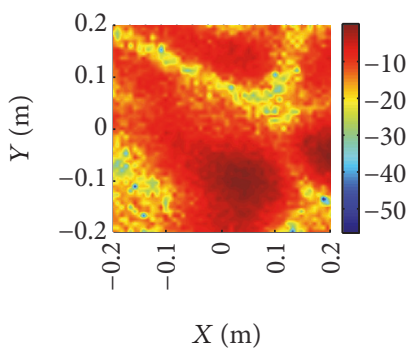

(h)

FIGURE 14: Field characteristics of two OAM modes under the first and second setup. (a, e) and (b, f) refer to the field characteristics when producing OAM modes 1 and 2 in the aligned system, while $(c, g)$ and $(\mathrm{d}, \mathrm{h})$ are the field characteristics when producing OAM modes 1 and 2 in the misaligned system. (a, b, c, d) refer to the phase profiles and (e, f, g, h) correspond to the intensity patterns.

good orthogonal property. From the comparison, it can be concluded that the OAM steering technique with small elevation angle can preserve good orthogonality of OAM mode.

\section{Conclusions}

In this study, a radio OAM steering technique based on a UCA working at Ka-band was elaborated to help solve the misalignment for OAM-based system. The performance of the radio OAM steering technique was then conducted. Simulation results showed that the degradation and contamination problems of OAM mode would occur when the steering elevation angle is large. And the higher OAM mode suffers from more severe distortion problem, which increases the BER of communication to some extent. To experimentally analyze the performance of radio OAM steering, UCAs with formulated feeding network were designed. The measurement results exhibited the significant distortion of twisted beam when the configuration of the system was in a misaligned condition, compared to the aligned configuration. Experiments also showed that good quality of OAM modes can be regained in spite of minor distortions for misaligned system, verifying the feasibility of the proposed OAM steering technique. In addition, influence of radio OAM steering technique on OAM modes and transmission performance and the mismatched polarization problem were experimentally evaluated, in a good agreement with the simulation results. The design of the feeding network based on a UCA to realize twisted beams steering technique is beneficial for the misalignment solution of OAM-based system. 


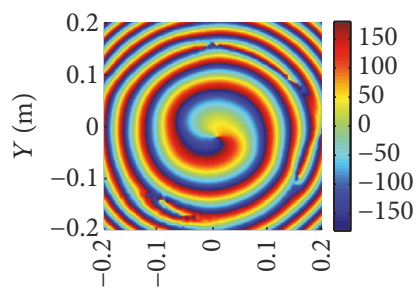

$X(\mathrm{~m})$

(a)

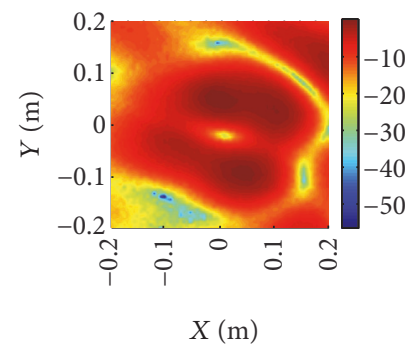

(e)

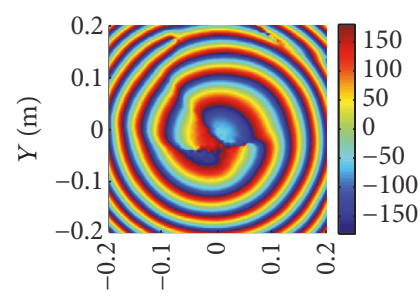

$X(\mathrm{~m})$

(b)

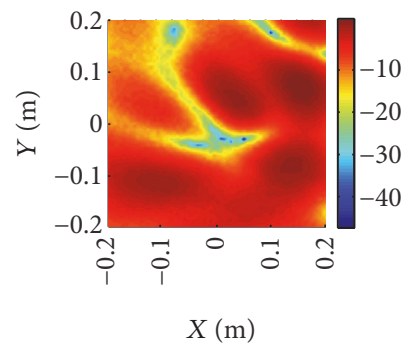

(f)

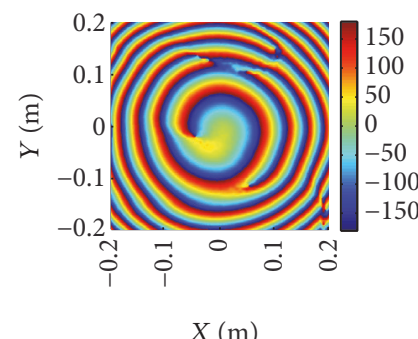

(c)

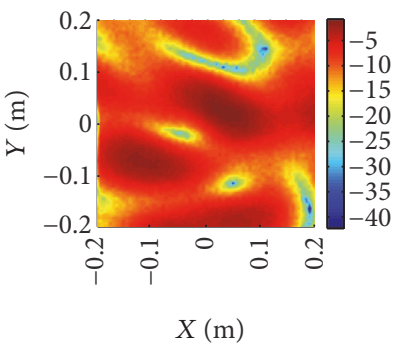

(g)

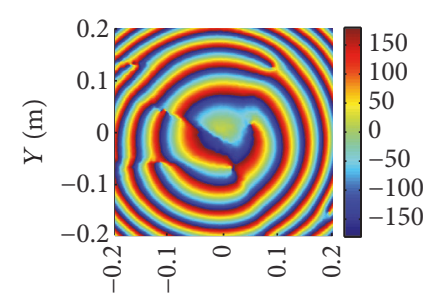

$X(\mathrm{~m})$

(d)

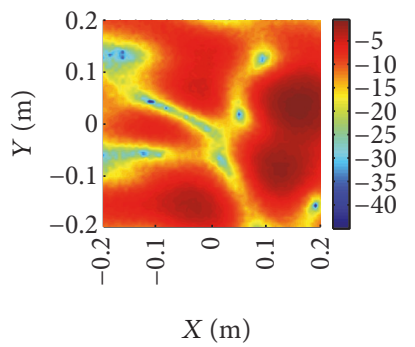

(h)

FIgURE 15: Field characteristics of two OAM modes under the third setup with different polarization probes. (a, e) and (b, f) refer to the field characteristics of OAM modes 1 and 2 measured with horizontal polarization probe, while $(\mathrm{c}, \mathrm{g})$ and $(\mathrm{d}, \mathrm{h})$ are the field characteristics of OAM modes 1 and 2 measured with the vertical polarization probe.

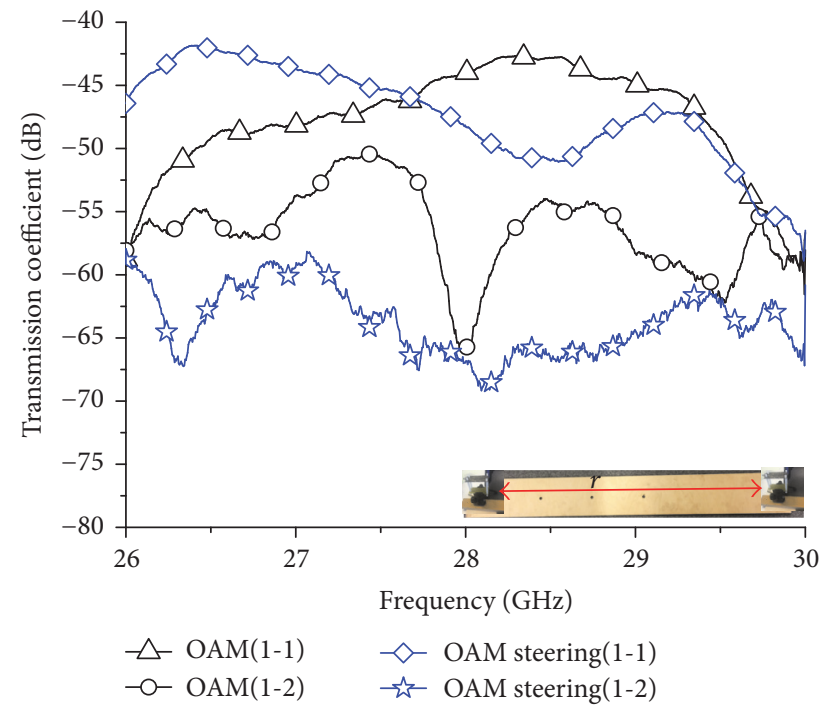

FIGURE 16: Transmission coefficients using nonsteering and steering OAM waves.

\section{Conflicts of Interest}

The authors declare that they have no conflicts of interest.

\section{References}

[1] L. Allen, M. W. Beijersbergen, R. J. C. Spreeuw, and J. P. Woerdman, "Orbital angular momentum of light and the transformation of Laguerre-Gaussian laser modes," Physical Review A, vol. 45, no. 11, pp. 8185-8189, 1992.
[2] J. P. Torres and L. Torner, "Twisted Photons: Applications of Light with Orbital Angular Momentum," Twisted Photons: Applications of Light with Orbital Angular Momentum, 2011.

[3] A. M. Yao and M. J. Padgett, "Orbital angular momentum: origins, behavior and applications," Advances in Optics and Photonics, vol. 3, no. 2, pp. 161-204, 2011.

[4] B. Thidé, H. Then, J. Sjöholm et al., "Utilization of photon orbital angular momentum in the low-frequency radio domain," Physical Review Letters, vol. 99, no. 8, Article ID 087701, 2007.

[5] S. M. Mohammadi, L. K. S. Daldorff, J. E. S. Bergman et al., "Orbital angular momentum in radio-a system study," IEEE Transactions on Antennas and Propagation, vol. 58, no. 2, pp. 565-572, 2010.

[6] A. Tennant and B. Allen, "Generation of OAM radio waves using circular time-switched array antenna," Electronics Letters, vol. 48, no. 21, pp. 1365-1366, 2012.

[7] F. Spinello, C. G. Someda, R. A. Ravanelli et al., "Radio channel multiplexing with superpositions of opposite-sign OAM modes," AEU - International Journal of Electronics and Communications, vol. 70, no. 8, pp. 990-997, 2016.

[8] R. Niemiec, C. Brousseau, K. Mahdjoubi, O. Emile, and A. Menard, "Characterization of an OAM flat-plate antenna in the millimeter frequency band," IEEE Antennas and Wireless Propagation Letters, vol. 13, pp. 1011-1014, 2014.

[9] L. Cheng, W. Hong, and Z.-C. Hao, "Generation of electromagnetic waves with arbitrary orbital angular momentum modes," Scientific Reports, vol. 4, article 4814, 2014.

[10] L. Cheng, W. Hong, and Z. Hao, "Design and implementation of planar reflection spiral phase plate for beams with orbital angular momentum," IET Microwaves, Antennas \& Propagation, vol. 11, no. 2, pp. 260-264, 2017.

[11] F. Tamburini, E. Mari, A. Sponselli, B. Thidé, A. Bianchini, and F. Romanato, "Encoding many channels on the same frequency 
through radio vorticity: first experimental test," New Journal of Physics, vol. 14, Article ID 033001, 2012.

[12] Y. Yan, G. Xie, M. P. J. Lavery et al., "High-capacity millimetrewave communications with orbital angular momentum multiplexing," Nature Communications, vol. 5, article 4876, 2015.

[13] S. Zheng, X. Hui, X. Jin, H. Chi, and X. Zhang, "Transmission characteristics of a twisted radio wave based on circular traveling-wave antenna," Institute of Electrical and Electronics Engineers. Transactions on Antennas and Propagation, vol. 63, no. 4, part 2, pp. 1530-1536, 2015.

[14] X. Hui, S. Zheng, Y. Chen et al., "Multiplexed millimeter wave communication with dual orbital angular momentum (OAM) mode antennas," Scientific Reports, vol. 5, article 10148, 2015.

[15] O. Edfors and A. J. Johansson, "Is orbital angular momentum (OAM) based radio communication an unexploited area?" Institute of Electrical and Electronics Engineers. Transactions on Antennas and Propagation, vol. 60, no. 2, part 2, pp. 1126-1131, 2012.

[16] M. Tamagnone, C. Craeye, and J. Perruisseau-Carrier, "Comment on 'Encoding many channels on the same frequency through radio vorticity: First experimental test"' New Journal of Physics, vol. 14, Article ID 118001, 2012.

[17] W. Zhang, S. Zheng, X. Hui et al., "Mode Division Multiplexing Communication Using Microwave Orbital Angular Momentum: An Experimental Study," IEEE Transactions on Wireless Communications, vol. 16, no. 2, pp. 1308-1318, 2017.

[18] K. Liu, Y. Cheng, Z. Yang, H. Wang, Y. Qin, and X. Li, “Orbitalangular-momentum-based electromagnetic vortex imaging," IEEE Antennas and Wireless Propagation Letters, vol. 14, pp. 711714, 2015.

[19] T. Yuan, H. Wang, Y. Cheng, and Y. Qin, "Electromagnetic Vortex-Based Radar Imaging Using a Single Receiving Antenna: Theory and Experimental Results," Sensors, vol. 17, no. 3, p. 630, 2017.

[20] G. Guo, W. Hu, and X. Du, "Electromagnetic vortex basedradar target imaging," " (in Chinese) J. Nat. Univ. Defense Technol, vol. 35, no. 6, p. 6, 2013.

[21] M. Lin, Y. Gao, P. Liu, and J. Liu, "Super-resolution orbital angular momentum based radar targets detection," Electronics Letters, vol. 52, no. 13, pp. 1168-1170, 2016.

[22] M. Lin, Y. Gao, P. Liu, and J. Liu, "Improved OAM-Based Radar Targets Detection Using Uniform Concentric Circular Arrays," International Journal of Antennas and Propagation, vol. 2016, Article ID 1852659, 2016.

[23] S. Yu, L. Li, G. Shi, C. Zhu, and Y. Shi, "Generating multiple orbital angular momentum vortex beams using a metasurface in radio frequency domain," Applied Physics Letters, vol. 108, no. 24, Article ID 241901, 2016.

[24] A. Bennis, R. Niemiec, C. Brousseau, K. Mahdjoubi, and O. Emile, "Flat plate for OAM generation in the millimeter band," in Proceedings of the 7th European Conference on Antennas and Propagation (EuCAP '13), pp. 3203-3207, Gothenburg, Sweden, April 2013. 


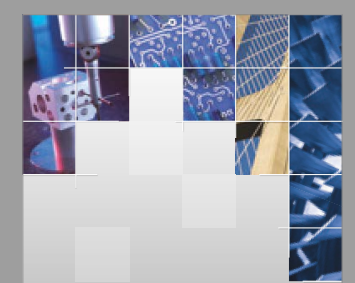

\section{Enfincering}
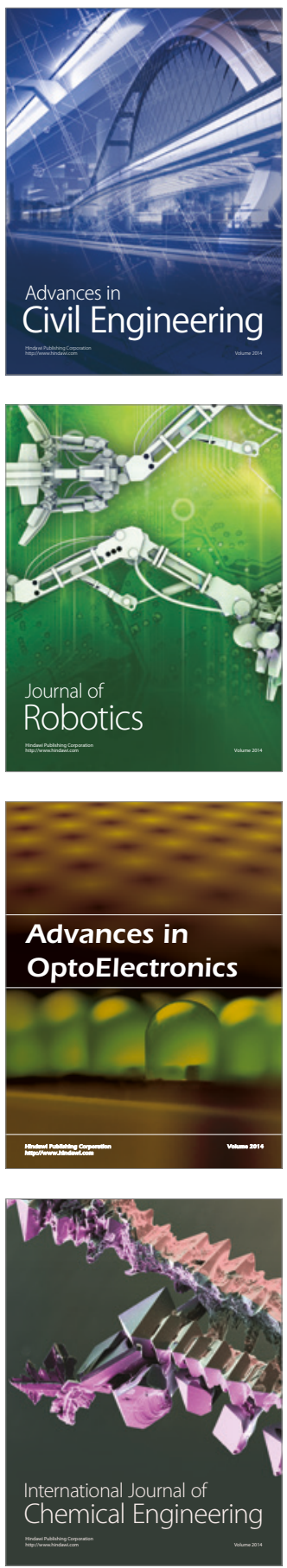

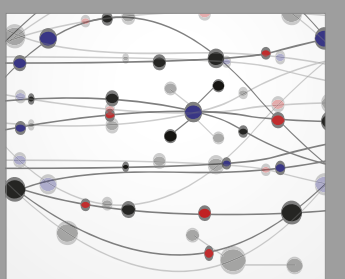

The Scientific World Journal

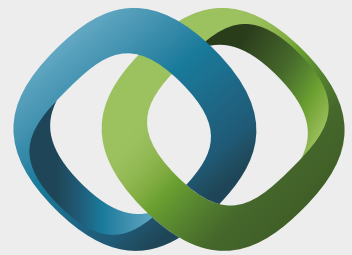

\section{Hindawi}

Submit your manuscripts at

https://www.hindawi.com
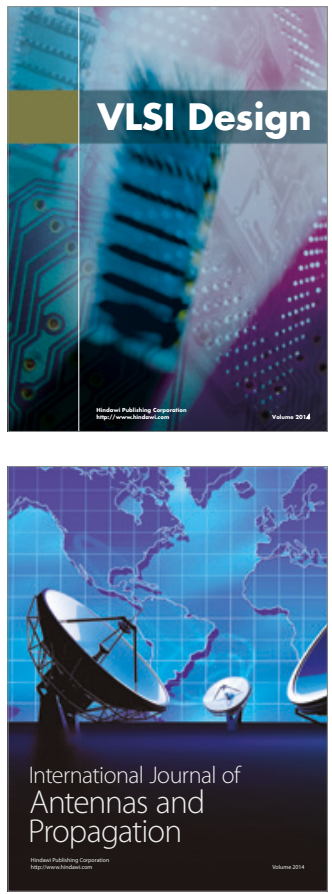

\section{Rotating}

Machinery
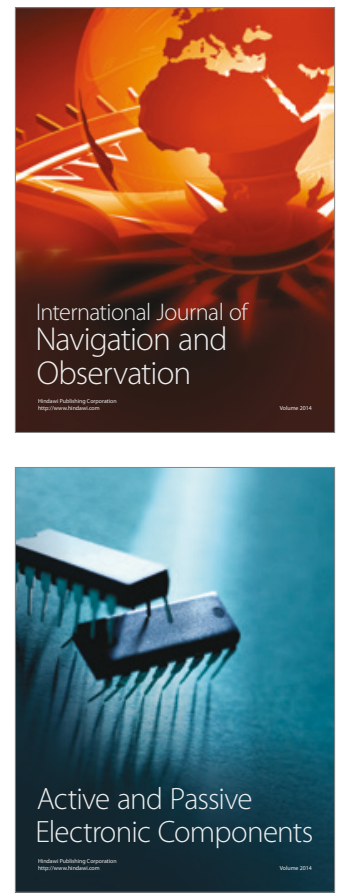
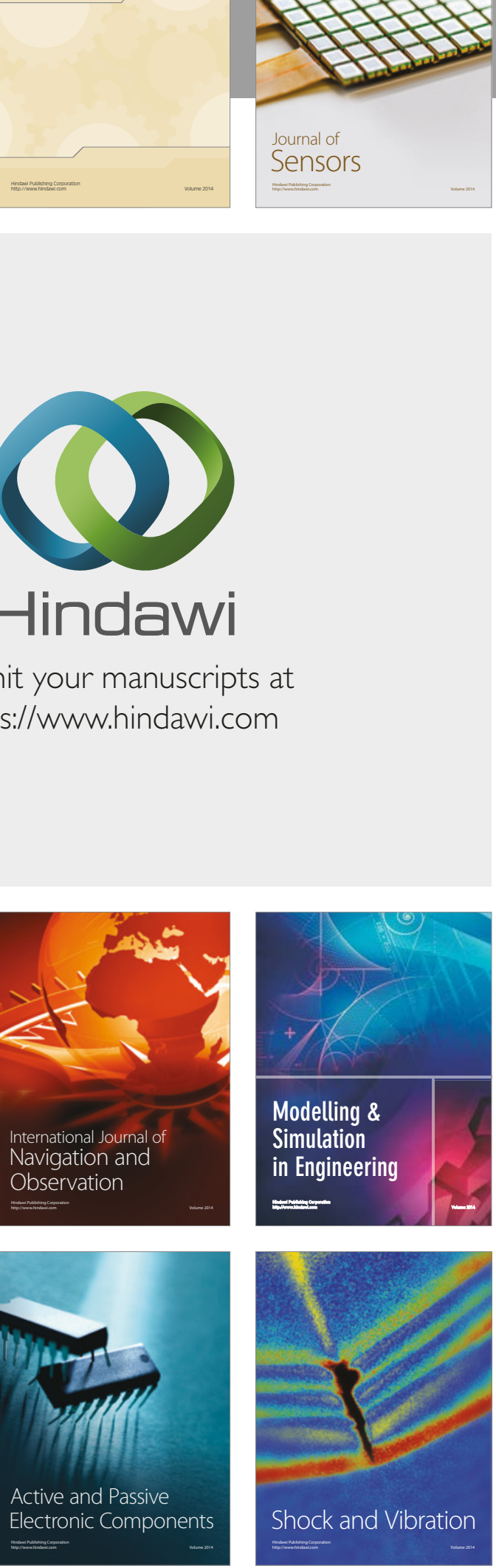
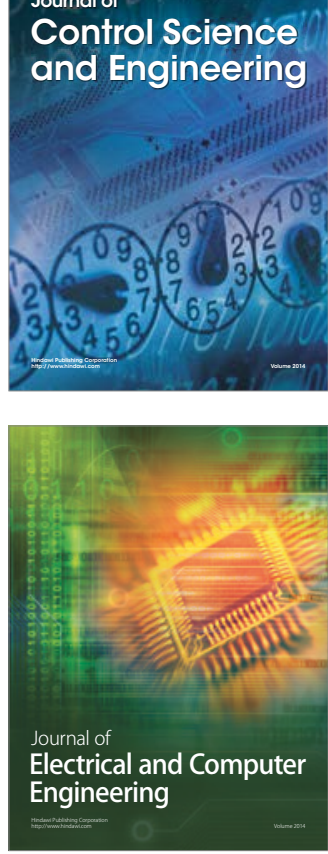

Distributed

Journal of

Control Science

and Engineering
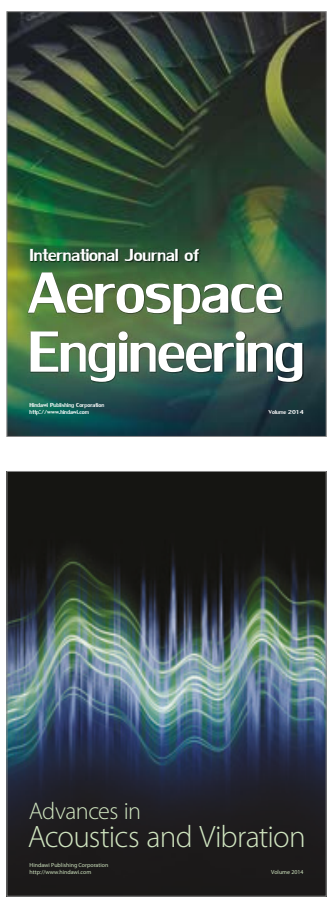

Sensor Networks 